\title{
LA CONSTRUCCIÓN TRADICIONAL EN LA ARQUITECTURA DE GAUDÍ
}

\author{
(TRADITIONAL CONSTRUCTION IN THE ARCHITECTURE OF GAUDI)
}

Juan Bassegoda Nonell, Director de la Cátedra Gaudi de la Escuela de Arquitectura de Barcelona de la Universidad Politécnica de Cataluña. España

\section{RESUMEN}

Para crear las originales y bellas formas arquitectónicas que han hecho famoso a Gaudi, el arquitecto se limitó a utilizar los materiales (ladrillo manual, piedra, cerámica vidriada, morteros de cal y de cemento rápido, yeso, etc.) y las técnicas tradicionales, o técnicas derivadas de ellas. El autor de este articulo pasa revista a estas técnicas constructivas gaudinianas, desde el pilar sencillo a los paraboloides hiperbólicos, pasando por el pilar cartabón, el falso arco, arco de festón o perfiles de catenaria, arcos de rosca, bóvedas tabicadas, bóvedas nervadas, cúpulas, pechinas, arcos tabicones, etcétera.
El principal mérito de Gaudi, además de su ingenua inspiración artística que lo hizo tan amado de las Musas, es el de haber creado formas nuevas e inéditas utilizando los materiales y las técnicas tradicionales. Por esta razón la arquitectura de Gaudí es intemporal, pudo haberla hecho tal cual en el siglo XIV o en los siglos futuros.

La construcción gaudiniana se hizo a base de piedra, ladrillo manual, cemento rápido, yeso y mortero de cal. No hizo nunca hormigón armado y el cemento portland lo empleó solamente para enlucidos. Por lo que se refiere a materias primas Gaudí usó distintos tipos de ladrillo hecho a mano -el ladrillo común, el mediano, la rasilla, el picholín, el prensado o el ladrillo recocho-, mortero de cal, cemento rápido y yeso. Con tan antiguos y acreditados elementos Gaudí se las ingenió para producir formas que, en su tiempo, chocaron por su originalidad. Unos ejemplos ilustrarán el aserto.

\section{SUMMARY}

In order to create the original and beautiful architectural forms which made Gaudi famous, the architect solely employed traditional materials (brick, stone, glazed ceramic lime and quick-drying cement for mortar, plaster, etc.) and techniques, or their derivations. The author reviews these Gaudinian construction techniques, from the simple column to hyperbolic paraboloids, the square column, the false arch, festoon arches or catenary profiles, spiral arches, Catalan vaults (brick vault of one, two or more ceilings of brick laid flat), ribbed vaulting, cupolas, penditives, partition arches, etc.

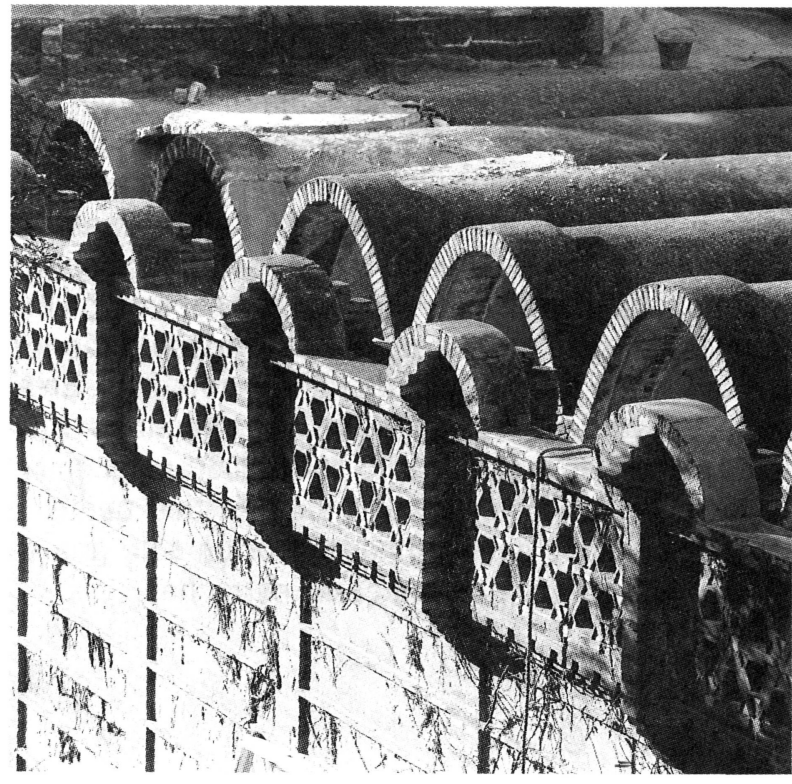

Fig. 1.-Bóvedas tabicadas de perfil catenárico de Tâs caballerizas Güell de Barcelona, sede actual de la Cátedra Gaudi (1884-1887). (Foto Cátedra Gaudi). 
En el Colegio Teresiano de la calle Ganduxer de Barcelona (1888-1889) contruyó pilares de dos metros de altura con fábrica de ladrillo visto formada por la superposición de ladrillos picholines. La base eran tres piezas iguales, lo mismo que el capitel, y el fuste la superposición de 26 picholines que miden tan sólo $29 \times 10 \times 5 \mathrm{~cm}$.

Otra forma singular era la que el profesor Fernández Casado llamaba el pilar cartabón, o pilastra cuyas hiladas horizontales de ladrillo avanzan un tanto sobre las inmediatas inferiores, formando un pilar inclinado o ménsula de gran canto. El perfil inclinado que se genera es recto, si el avance de cada hilada es uniforme, si el saledizo aumenta a medida que gana altura, se tiene perfil curvo, sensiblemente catenárico. Ejemplos de pilar cartabón se hallan en las galerías del picadero de la Finca Güell (1884-1887), en las tribunas de la casa Vicens (1883-1888), o en las esquinas del Colegio Teresiano. La combinación de dos pilares cartabón contrapuestos genera el falso arco. El encuentro se salda con una hilada horizontal o bien con un corto arco a sardinel. Es el caso de la arquería de la casa del portero en la finca Güell, la planta baja del colegio citado, etcétera.

El paso siguiente en el sistema de arcos empleado por Gaudi lo forman los perfiles de catenaria. Una cadena de eslabones iguales suspendida por sus extremos forma una curva espontánea, llamada catenaria o festón, y es tal que su perfil sigue exactamente la línea de tensiones, por la que pasan las fuerzas resultantes de una estructura extendida o estirada. La forma conjugada de ésta, es decir la simétrica, respecto al eje horizontal, define el perfil del arco catenárico que sigue la forma de la línea de presiones de una estructura que trabaja comprimida.

Gaudi usó de esta forma en muchos casos con excelentes resultados mecánicos y estéticos. En la cabaIleriza de la Finca Güell, ahora "Aula Discretorum" de la Cátedra Gaudí, hay una combinación de dos pilares cartabón contrapuestos unidos en su tercio medio por un arco de festón. Son famosos los falsos arcos del pasillo del primer piso del Colegio Teresiano, hechos de simple ladrillo picholín, con escasa separación entre ellos, lo que permite suprimir el forjado superior, que se reduce a una solera de tablero cerámico. En el primer desván y en los corredores que circundan el patio de parcela de la casa Batlló (1904-1906) aparecen estos arcos catenáricos, muy delgados, hasta el punto de ser tabicones, es decir formados por un solo grueso de ladrillos unidos por sus caras estrechas.

Fig. 3.-Falso arco de la fachada principal del Colegio Teresiano de Barcelona. (Dibujo de Juan Bergós Massó, arquitecto).

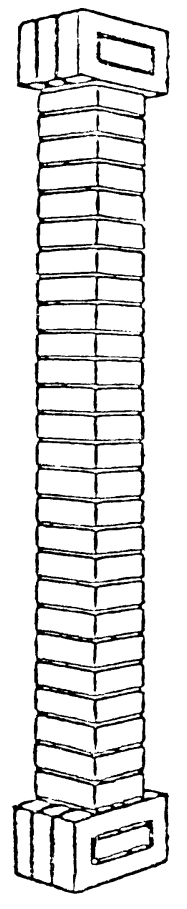

Fig. 2.-Pilar de ladrillo picholín. Primer piso del Colegio Teresiano. Barcelona (1888-1889).

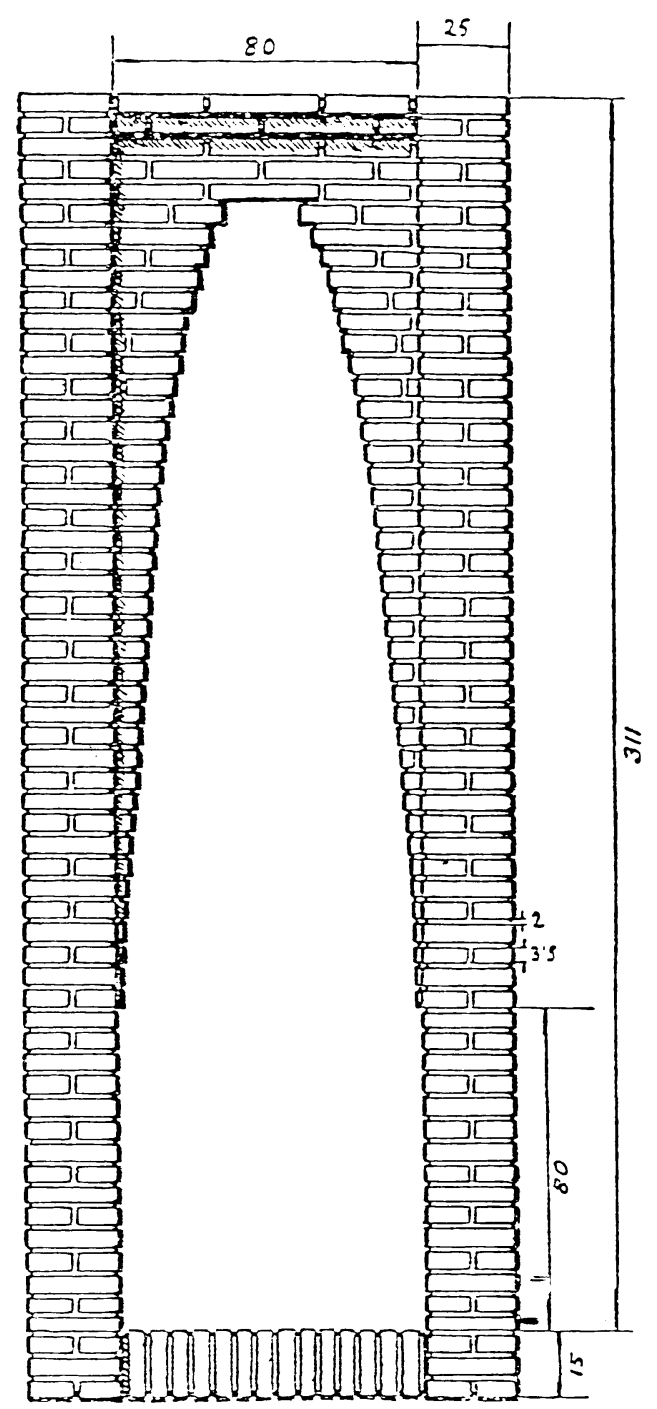

http://informesdelaconstruccion.revistas.csic.es 
El más sonado de los casos de conjunto de arcos tabicones se halla en el desván de la Pedrera (1906-1912). En la planta noble del palacio de los Güell y en las Bodegas Güell de Garraf (1895-1897), había usado la piedra en vez de fábrica de ladrillo para realizar esta clase de arcos.

Otro tipo de arco usado por Gaudí es el de rosca formado con ladrillos asardinados y poco canto. Puede hacerse con todo tipo de ladrillos y se recibían con cemento rápido o mortero de cal. Se forma con distintas hiladas de ladrillo a matajuntas. La primera rosca se suele tomar con cemento rápido y las demás con mortero de cal. El cemento rápido tiene una parte de arcilla por dos y media de caliza, que se cuecen a temperatura inferior a la de vitrificación.

Ejemplos de arcos de rosca figuraban en la cascada del jardín de la casa Vicenç (1883), en el primer desván de Bellesguard (1900-1909) (con ladrillo picholín) y entre las columnas del templo dórico o sala hipóstila del Park Güell (1909-1910). En algunos casos Gaudí consolidó este tipo de arcos usando tirantes de hierro que absorbieran la coz. El tirante de hierro dulce, redondo o en T, se amarra en las testas acarteladas del arco. Así se podía ver en la ampliación del taller de la Sagrada Familia (1897) y en el entresuelo de Bellesguard, donde los tirantes tienen forma helicoidal.

Donde Gaudi creó un mundo de estructuras y formas, muchas de ellas inéditas en arquitectura, fue en el campo de las bóvedas tabicadas. Estas estructuras - tan habituales en Cataluña, aunque comunes igualmente en el Rosellón y en Castilla- se forman con ladrillos o rasillas colocados por tabla. La primera hoja o sencillo se hace humedeciendo moderadamente las piezas que se toman con yeso o cemento rápido, según sean interiores o exteriores. El albañil prepara las piezas y el peón las lardea con el aglomerante por sus caras estrechas y las entregas al albañil que las coloca en obra y las afianza con un certero golpe al sesgo con el canto del palustre en su sitio y recoge los mocarros o rebabas. Luego se coloca el doblado a torta y restregón. Con bóvedas tabicadas se hacen en Cataluña escaleras de elegantes y atrevidas formas sin apoyos laterales.

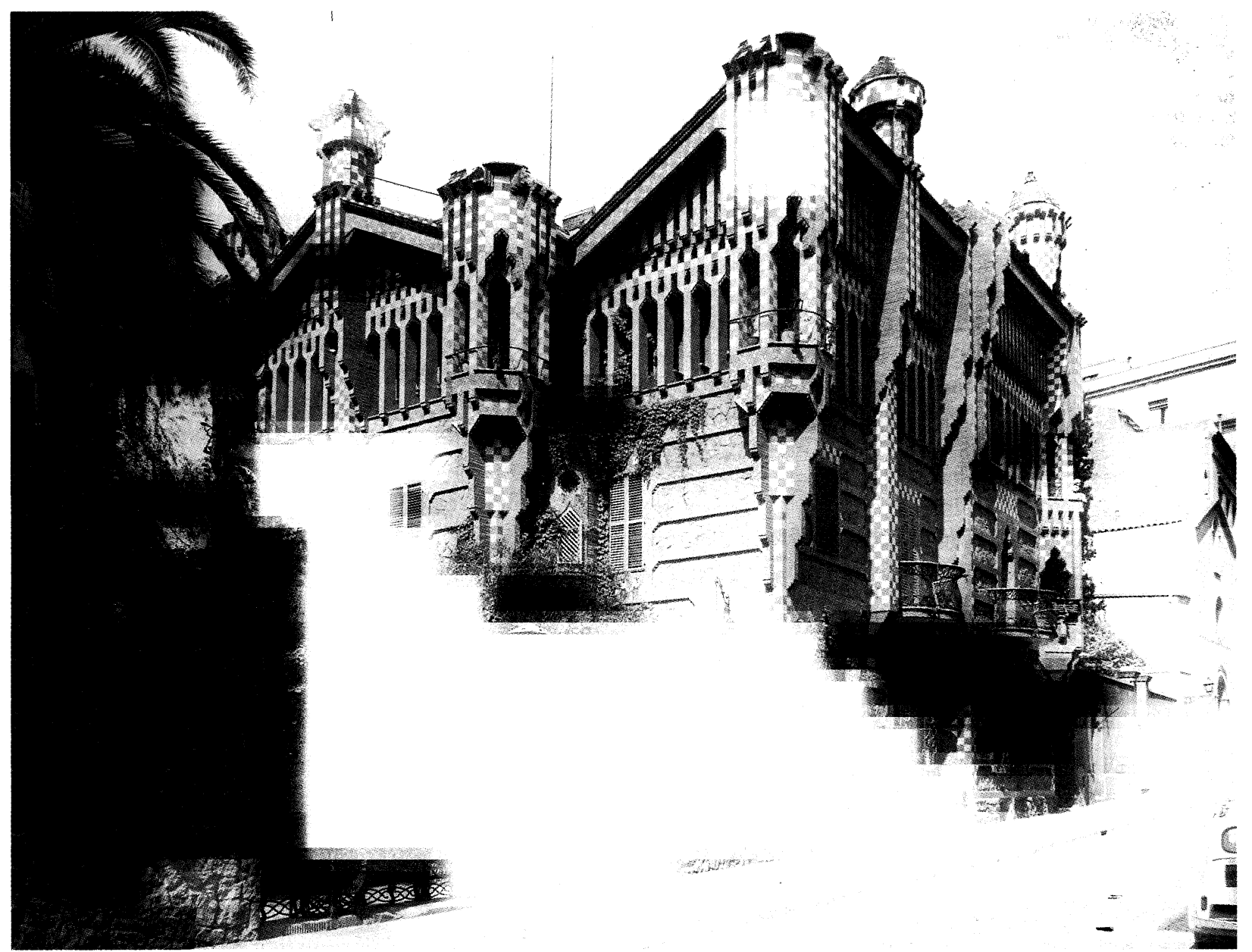

Fig. 4.-Casa Vicens de Barcelona. Tribunas angulares de pilar cartabón. (1883-1888). (Foto Amics de Gaudí). 


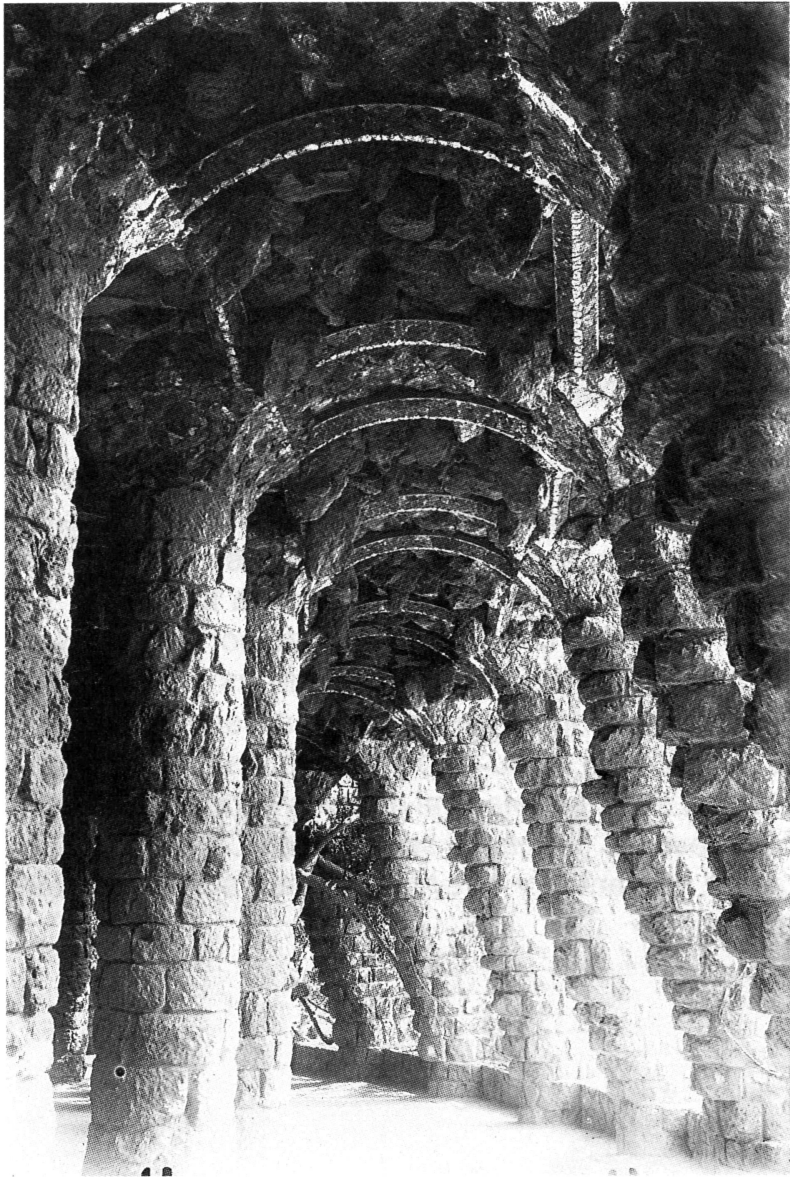

Fig. 5.-Nervios prefabricados, bóvedas tabicadas y falsa bóveda de piedra rústica en el pont del mig del Park Güell de Barcelona. (Foto Cátedra Gaudi, septiembre de 1921).

El tipo más sencillo de bóveda tabicada es el de cañón seguido que se construye con una única cercha corrediza que se usa sólo para tender el sencillo, que sirve de cercha del doblado. De este tipo las hay en el semisótano de Bellesguard, de perfil escarzano o entre los falsos arcos de la caballeriza Güell, aquí de perfil catenárico. Las formas usuales de las bóvedas tabicadas, además de las propias de las escaleras, son las de cañón seguido por arista esquifada de rincón de claustro y las cúpulas hemiesféricas, acuminadas o por sectores, o gajos, de las cuales se derivan otras formas ideadas por Gaudí.

En el picadero de la Finca Güell construyó una cúpula en forma de hiperboloide de revolución y, en la linterna de la misma, otra en forma de catenoide o catenaria girando sobre su eje vertical. En el refugio de coches en la entrada del Park Güell hay una bóveda tabicada en forma de hiperboloide, recubierta de piedra.

Donde más singular se presenta el uso de bóvedas tabicadas en la obra de Gaudí es cuando se tornan paraboloides hiperbólicos. Gaudí utilizó su forma más sencilla, es decir, la que se obtiene con dos directrices rectas, no paralelas en el espacio, sobre las que descansan las generatrices, igualmente rectas. Resulta asi una superficie alabeada en el espacio, compuesta totalmente por líneas rectas y por tanto fácil de construir por los albañiles, a los que únicamente se debe dar la situación de las directrices para que sobre ellas vaya deslizando la regla o el cordel que cada vez definirá una generatriz.

El más notable ejemplo de este sistema es el de los muros y bóvedas de la cripta de la Colonia Güell. Todos los muros tienen esta forma alabeada reglada son de ladrillo, en parte recubierto con piedra volcánica. En el porche las bóvedas son tabicadas y en forma de paraboloide hiperbólico, con decoración de cerámica vidriada. De este tipo deberán ser las de la nave mayor de la Sagrada Familia, aunque está aún por decidir el material a emplear. Nadie había utilizado estas formas en las bóvedas antes que Gaudi y el efecto es sorprendente ya que se muestran con el intradós convexo, al revés de lo que sucede con las demás bóvedas y cúpulas.

Con los paraboloides hiperbólicos de la Colonia Güell puede decirse que Gaudí inauguró una nueva estética de las bóvedas, pero en realidad fue bastante más lejos ya que, convencido de las cualidades plásticas de este tipo de fábrica, lo convirtió de arquitectura, en escultura decorativa.

También construyó Gaudi bóvedas tabicadas en forma de triángulo alabeado o pechina en la Finca Güell como soporte de la cúpula del picadero. En las chimeneas de la azotea del palacio del conde de Güell (1886-1888), se compusieron formas decorativas muy diversas, algunas de ellas también formadas con bóveda tabicada. En la casa Batlló, las chimeneas se hicieron totalmente con esta técnica, utilizando fragmentos de rasilla en vez de piezas enteras para conseguir las más complejas formas. Tal proceder culmina en las chimeneas de la Pedrera, de las más variadas, elegantes y bellas formas.

De tales chimeneas es ya interesante el diseño o proyecto, que no se hacía dibujando, sino con maquetas de yeso a escala 1:10. José Bayó Font (1878-1971), contratista de las casas Batlló i Milà, relató cómo se elaboraron las maquetas en la propia obra por el yesero modelista Juan Bertran y los oportunos retoques de mano del propio Gaudí. Una maqueta de chimenea sencilla, otra doble y la del famoso ventilador de las lemniscatas alabeadas, se han conservado y figuran en la colección de arquitectura de la Cátedra Gaudí. A partir de la maqueta, los albañiles forjaban las bóvedaschimeneas directamente, por el simplè sistema de sacar puntos. Una vez terminadas las formas eran revocadas con mortero de cal. 
En la azotea de la casa Milá, además de las chimeneas, de los afamados guerreros que han sido objeto de poesías y leyendas, y de los ventiladores, hay ocho salidas de escalera y estructuras complejas muy interesantes. Para salvar el desnivel entre el suelo del desván y la solera de la azotea se construyó un cilindro tabicado al que se enrolla el helicoide de la escalera, igualmente tabicada, que se cierra exteriormente por otro tabique en forma de tronco de cono, que emerge sobre la azotea.

Ortogonalmente al eje del cono y en su parte exterior, por encima de la solera de la terraza, se construyeron tabiques verticales en disposición radial. Sobre tales tabiques los ayudantes de Gaudí, José Canaleta Cuadras y Domingo Sugrañes Gras, trazaban unas líneas curvas para que el albañil demoliera la parte del tabique, de las líneas para afuera. Entre las líneas curvas resultantes en los extremos de los tabiques se tendían nuevas bóvedas tabicadas, que formaban el caparazón exterior de la salida de escalera. Estas elegantes superficies eran luego aplacadas con cerámica troceada o con fragmentos de losetas de mármol o pedazos de loza. Naturalmente el origen de tales formas era una maqueta de yeso previamente elaborada por Gaudí y Bertran.

Los acabados de tales superficies eran de diversos tipos. El más sencillo era el revocado con mortero de cal, sigue el aplacado con azulejos troceados (el conocido trencadís), aplacado con azulejos enteros en superficies planas, revestimiento vignolesco con pequeñas piedras sin desbastar, revestimiento rústico de piedras de regular tamaño imitando una mampostería ordinaria, revestimiento con cristales de colores o revestimiento con cristales transparentes pintados de color por su cara interna. Del primer tipo se tienen las chimeneas de la casa Milà, del segundo el alicatado con piezas hexagonales vidriadas del intradós de la cúpula interior del palacio Güell. Del aplacado con azulejos troceados hay múltiples ejemplos en la Finca Güell, en el parque Güell, cripta de la Colonia Güell y otros edificios del arquitecto. El revestimiento de piedras menudas se encuentra en los contrafuertes del porticado del parque Güell; con piedras rústicas de regular tamaño, en los viaductos del mismo parque; el revestimiento de cristales de colores, en la fachada de la casa Batlló y los cristales pintados, en la torre de Bellesguard.

Otro tipo de bóvedas tabicadas inventado por Gaudí fue el de conoide de plano director, superficie reglada alabeada cuyas generatrices resbalan sobre tres curvas en el espacio. Una de las curvas puede reducirse a una recta, situada en el infinito, que equivale a un plano, que se denomina director. Cuando las directrices del conoide son paralelas al plano director y resbalan sobre las dos curvas directrices, se obtiene el cilindroi-

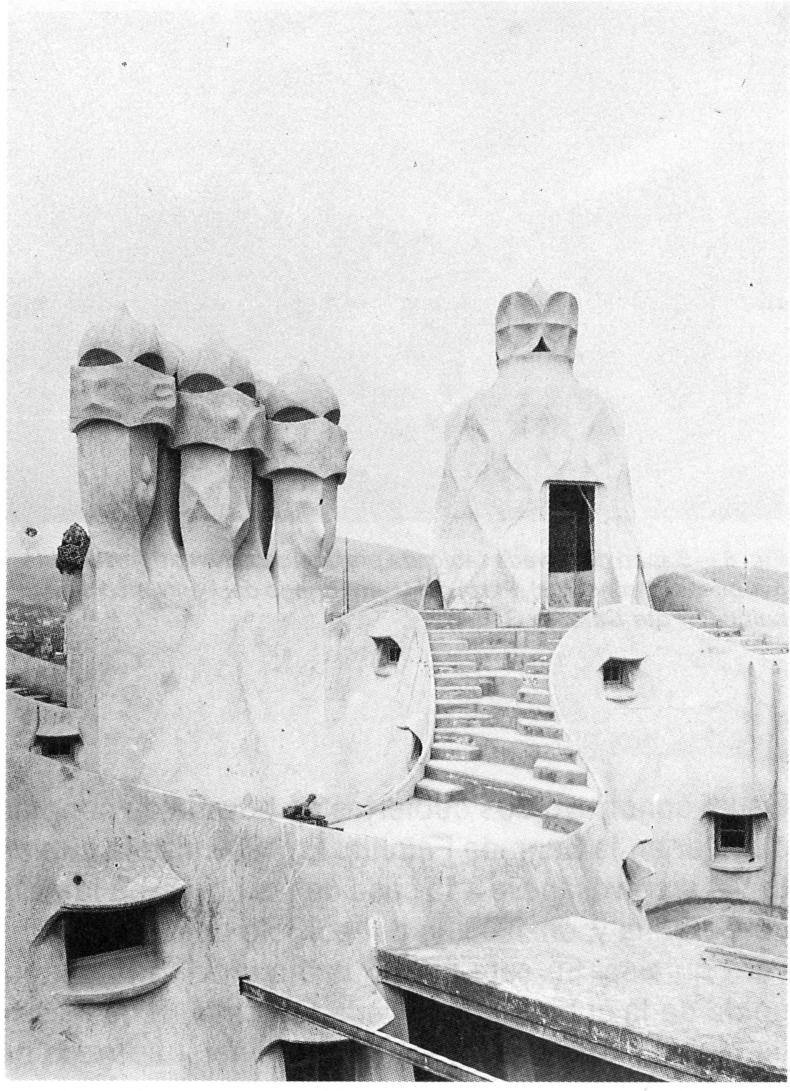

Fig. 6.-Chimeneas y salida de escalera de bóveda tabicada en la azotea de la Casa Milà de Barcelona (1906-1912). (Foto Cátedra Gaudi).

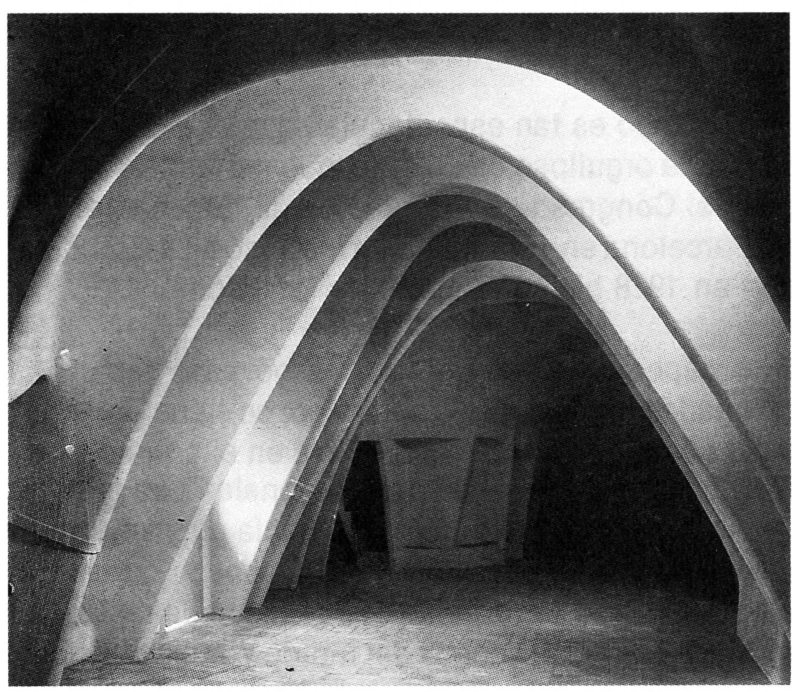

Fig. 7.-Arcos catenáricos en el primer desván de la Casa Batlló de Barcelona (1904-1906). (Foto Cátedra Gaudi, 1907).

de de plano director. Si una de las curvas directrices se transforma en una recta, se obtiene el conoide de plano director. El paraboloide hiperbólicō es un cilindroide con dos directrices rectas. 


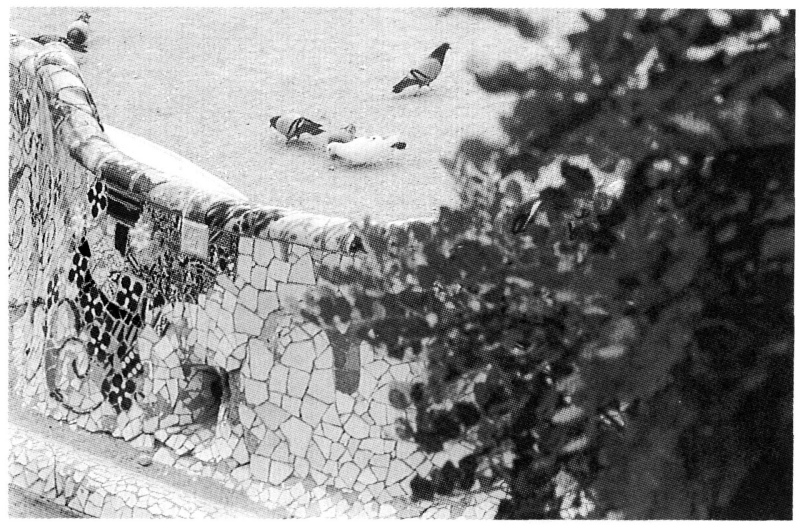

Fig. 8.-Banco de bóveda tabicada prefabricada revestida de azulejo troceado (trencadis). Plaza del teatro griego del Park Güell de Barcelona. (Foto Cátedra Gaudí).

Gaudí construyó dos cubiertas de bóveda de conoide director en la Sagrada Familia. En 1906 hizo la cubierta del archivo, anexo a la casa de las obras, en la calle de Cerdeña y, en 1909, la del edificio de las Escuelas Parroquiales. En este último la directriz recta del conoide de la cubierta de bóveda tabicada, es la jácena de hierro situada en el eje longitudinal y apoyada en dos perfiles de hierro. Las generatrices son tablones de madera que apoyan alternativamente sobre el muro de fachada, cuya coronación es una alternancia de arcos de círculo en la jácena recta y en el muro opuesto, donde las ondulaciones son conjugadas de las del muro opuesto.

El resultado es tan espectacular, que el propio Gaudi se sentía orgulloso de su obra, que mostró a sus colegas del Congreso de Arquitectos de España, reunido en Barcelona en 1916. También interesó a Le Corbusier, que en 1928 hizo un apunte de las Escuelas.

Otro aspecto interesante de las bóvedas tabicadas, exornadas con troceado cerámico, se tiene en el proceso de prefabricación que utilizó en el parque Güell. Para hacer los medallones hexagonales que adornan la escalera con sus azulejos, se hacía previamente un molde de madera, con la forma alabeada correspondiente. Encima se forjaban dos gruesos de rasilla con mortero de cal, se sacaba del molde y, en la cara junto a la madera, se aplacaba el azulejo troceado. Igual pro- cedimiento se siguió con el banco de la plaza del teatro griego que se prefabricó según dos moldes de curvatura opuesta, se aplacó la cerámica y se colocaron las partes en su sitio correspondiente. Es posible ver las juntas que tales encuentros originaron. Las columnas inclinadas de los viaductos, los nervios de las bóvedas de tales viaductos y los helicoides de la rampa peatonal, son también piezas de bóveda tabicada prefabricada y luego revestida con fragmentos de piedras, de mayor o menor tamaño.

Otro modelo original son los forjados sin vigas que se suplen con arcos tabicones rebajados, como sucede en el comedor de Bellesguard, o en los dormitorios de los pabellones de entrada en el parque Güell, donde Gaudí situó, ingenuamente, unos supuestos refuerzos de flejes retorcidos que, embebidos en mortero de cal, se oxidaron causando abundantes grietas.

Otro modelo único lo forman las bóvedas nervadas de Bellesguard, que no concurren en ninguna clave central y están hechas de ladrillo revocado con yeso. La variedad de soluciones empleadas por Gaudí, con estos sencillos medios, se completa con el soberbio tratamiento de la piedra que, en los campanarios de la Sagrada Familia, tomó forma de paraboloide de revolución y consigue un exquisito sentido de esbeltez, con la piedra trabajando solamente a compresión, siguiendo rigurosamente las líneas de presiones.

La relación podría ir mucho más allá de la extensión atribuida a este esbozo, por ello se cierra con una somera explicación de la técnica de cielos rasos tridimensionales de la casa Milà, con el normal sistema de tejido de cañizo y revoco de yeso blanco. En vez de colocar el cañizo plano, sujeto con clavos en listones del entrevigado, Gaudí mandaba colocar unas manguetas de madera, de longitudes diversas, donde se fijaba el cañizo que quedaba en forma tridimensional. Encima, los ayudantes trazaban unas curvas mediante clavos y el yesero terminaba la tarea dejando aquellas líneas nerviosamente curvadas, en resalto. Incluso se encuentran leyendas en estos resaltos llenas de sentido poético, como la del dormitorio del piso del señor Roca, donde se lee: "Oh Maria, no et sàpiga greu ser tan petita, perquè també ho són les flors i les estrelles" (Oh María no te duela ser tan pequeña porque también lo son las flores y las estrellas). Gaudí era así. 